\title{
STANDING WAVES \\ FOR NONLINEAR SCHRÖDINGER-POISSON EQUATION WITH HIGH FREQUENCY
}

\author{
JiAnQing Chen - ZhengPing Wang - XiaOju Zhang
}

Abstract. We study the existence of ground state and bound state for the following Schrödinger-Poisson equation

(P) $\left\{\begin{array}{l}-\Delta u+V(x) u+\lambda \phi(x) u=\mu u+|u|^{p-1} u, \quad x \in \mathbb{R}^{3}, \\ -\Delta \phi=u^{2}, \quad \lim _{|x| \rightarrow+\infty} \phi(x)=0,\end{array}\right.$

where $p \in(3,5), \lambda>0, V \in C\left(\mathbb{R}^{3}, \mathbb{R}^{+}\right)$and $\lim _{|x| \rightarrow+\infty} V(x)=\infty$. By using variational method, we prove that for any $\lambda>0$, there exists $\delta_{1}(\lambda)>0$ such that for $\mu_{1}<\mu<\mu_{1}+\delta_{1}(\lambda)$, problem (P) has a nonnegative ground state with negative energy, which bifurcates from zero solution; problem (P) has a nonnegative bound state with positive energy, which can not bifurcate from zero solution. Here $\mu_{1}$ is the first eigenvalue of $-\Delta+V$. Infinitely many nontrivial bound states are also obtained with the help of a generalized version of symmetric mountain pass theorem.

2000 Mathematics Subject Classification. Primary 35J20, 35J70.

Key words and phrases. Schrödinger-Poisson equation, ground states, bound states, variational method.

Jianqing Chen was supported by NSFC under grant No. 11371091 and the innovation group of 'Nonlinear analysis and its applications' (No. 021337120).

Zhengping Wang was supported by NSFC under grant No. 11271360. 


\section{Introduction}

In this paper, we consider the nonlinear Schrödinger-Poisson equation

$$
\left\{\begin{array}{l}
-i \frac{\partial \psi}{\partial t}-\Delta \psi+V(x) \psi+\lambda \phi(x) \psi=|\psi|^{p-1} \psi, \quad(t, x) \in \mathbb{R} \times \mathbb{R}^{3}, \\
-\Delta \phi=|\psi|^{2}, \quad \lim _{|x| \rightarrow+\infty} \phi(x)=0,
\end{array}\right.
$$

where $\psi: \mathbb{R} \times \mathbb{R}^{3} \rightarrow \mathbb{C}$ denotes the wave function, $\lambda$ is a positive parameter, $p \in(3,5)$, and $V, \phi$ are real valued functions and represent the effective potential and the electric potential, respectively. Problem (1.1) arises from semiconductor theory, see [9], [13] for more physical background.

Recently, there are many papers devoted to looking for standing wave solutions to problem (1.1), that is, $\psi(t, x)=e^{-i \mu t} u(x)$, where $u(x)$ is a real valued function and $\mu \in \mathbb{R}$ denotes the frequency. Then $u(x)$ satisfies the following stationary equation

$$
\left\{\begin{array}{l}
-\Delta u+V(x) u+\lambda \phi(x) u=\mu u+|u|^{p-1} u, \quad x \in \mathbb{R}^{3}, \\
-\Delta \phi=u^{2}, \quad \lim _{|x| \rightarrow+\infty} \phi(x)=0 .
\end{array}\right.
$$

See [2], [6], [7], [10]-[12], [17], [19] and the references therein for all kinds of $V$ and more general nonlinearities.

Throughout this paper, we assume that $V(x)$ satisfies the following condition

(V1) $V \in C\left(\mathbb{R}^{3}, \mathbb{R}^{+}\right)$and $\lim _{|x| \rightarrow+\infty} V(x)=\infty$.

Define

$$
H=\left\{u \in W^{1,2}\left(\mathbb{R}^{3}\right): \int_{\mathbb{R}^{3}} V(x) u^{2} d x<\infty\right\},
$$

with the norm

$$
\|u\|^{2}:=\int_{\mathbb{R}^{3}}\left(|\nabla u|^{2}+V(x) u^{2}\right) d x .
$$

It is known that, under the condition (V1), the embedding $H \hookrightarrow L^{q}\left(\mathbb{R}^{3}\right)(2 \leq$ $q<6)$ is compact. Moreover, there is a sequence of eigenvalues $\left(\mu_{n}\right)$ of $-\Delta+V$ in $H$ such that $0<\mu_{1}<\mu_{2} \leq \mu_{3} \leq \ldots \leq+\infty$ and $H=\operatorname{span}\left\{e_{j}: j \geq 1\right\}$, where $e_{j}$ is the corresponding eigenfunction to $\mu_{j}$ with $\left\|e_{j}\right\|=1$.

For $u \in H$, we denote the unique solution of $-\Delta \phi=u^{2}$ in $D^{1,2}\left(\mathbb{R}^{3}\right)$ by $\phi_{u}$, and

$$
\phi_{u}(x)=\frac{1}{4 \pi} \int_{\mathbb{R}^{3}} \frac{u^{2}(y)}{|x-y|} d y .
$$

Then equation (1.2) can be rewritten as

$$
-\Delta u+V(x) u+\lambda \phi_{u}(x) u=\mu u+|u|^{p-1} u, \quad x \in \mathbb{R}^{3} .
$$


Define the energy functional $I_{\mu}: H \rightarrow \mathbb{R}$ by

$$
\begin{aligned}
I_{\mu}(u)=\frac{1}{2} \int_{\mathbb{R}^{3}}\left(|\nabla u|^{2}+(V(x)-\right. & \left.\mu) u^{2}\right) d x \\
& +\frac{\lambda}{4} \int_{\mathbb{R}^{3}} \phi_{u} u^{2} d x-\frac{1}{p+1} \int_{\mathbb{R}^{3}}|u|^{p+1} d x .
\end{aligned}
$$

Then, $I_{\mu} \in C^{1}(H, \mathbb{R})$ and for any $\varphi \in H$ we have that

$$
\begin{aligned}
\left\langle I_{\mu}^{\prime}(u), \varphi\right\rangle=\int_{\mathbb{R}^{3}}(\nabla u \nabla \varphi+(V(x)-\mu) u \varphi) d x & \\
& +\lambda \int_{\mathbb{R}^{3}} \phi_{u} u \varphi d x-\int_{\mathbb{R}^{3}}|u|^{p-1} u \varphi d x .
\end{aligned}
$$

If $u \in H \backslash\{0\}$ and $\left\langle I_{\mu}^{\prime}(u), \varphi\right\rangle=0$ for all $\varphi \in H$, we say that $u$ is a bound state of (1.4). Furthermore, a function $u_{0}$ is called a ground state of (1.4) if $u_{0}$ is a bound state of $(1.4)$ and $I_{\mu}\left(u_{0}\right) \leq I_{\mu}(u)$ for any bound state $u$ of (1.4).

If $\mu<\mu_{1}$ in (1.4), we may define the following equivalent norm on $H$ by

$$
\|u\|_{\mu}^{2}:=\int_{\mathbb{R}^{3}}\left(|\nabla u|^{2}+(V(x)-\mu) u^{2}\right) d x .
$$

Then for any $\lambda>0, p \in(3,5)$, and under condition (V1), we can easily prove that (1.4) has a ground state by Nehari manifold method, see [20].

In this paper, we mainly study the existence of bound state, especially ground state, to equation (1.4) for $\mu \geq \mu_{1}$, which is the so called high frequency case in the title. First, we show that (1.4) has a nonnegative bound state with positive energy.

TheORem 1.1. Let $p \in(3,5), \lambda>0$ and (V1) holds. Then there exists $\delta(\lambda)>0$ such that for any $\mu \in\left[\mu_{1}, \mu_{1}+\delta(\lambda)\right)$, problem (1.4) has a nonnegative bound state $u_{1, \mu}$ with $I_{\mu}\left(u_{1, \mu}\right)>0$. Moreover, for any sequence $\mu^{(n)} \downarrow \mu_{1}$, there exists $u_{\mu_{1}} \in H$ with $I_{\mu_{1}}^{\prime}\left(u_{\mu_{1}}\right)=0$ and $I_{\mu_{1}}\left(u_{\mu_{1}}\right)>0$, such that $u_{1, \mu^{(n)}} \rightarrow u_{\mu_{1}}$ strongly in $H$.

On basis of Theorem 1.1, we may define the set of all bound states to (1.4):

$$
\mathcal{N}=\left\{u \in H \backslash\{0\}: I_{\mu}^{\prime}(u)=0\right\} \neq \emptyset .
$$

To get the existence of ground state to (1.4), we consider the following minimization problem

$$
c_{0}=\inf \left\{I_{\mu}(u): u \in \mathcal{N}\right\}
$$

If we can prove that $c_{0}>-\infty$ and $c_{0} \neq 0$, then by the compactness lemma (see Lemma 2.1) and solving the above minimization problem, the ground state to (1.4) can be obtained. But, it seems not obvious that $c_{0}>-\infty$ and $c_{0} \neq 0$. 
Here, let us recall that if $\lambda=0$ in (1.4), by the method of Szulkin and Weth [18] we can prove that $c_{0}>0$. Motivated by Pankov [14], see also [18], we introduce the set

$$
\mathcal{N}_{1}=\left\{u \in H \backslash H_{1}: I_{\mu}^{\prime}(u)=0\right\},
$$

where $H_{1}=\operatorname{span}\left\{e_{1}\right\}$. We first see that $\mathcal{N}=\mathcal{N}_{1}$, that is, $\mathcal{N}_{1}$ contains all bound states of (1.4). Otherwise, if there exists $u \in H_{1} \backslash\{0\}$ such that $I_{\mu}^{\prime}(u)=0$, then noting that $\lambda=0$ and $\mu>\mu_{1}$, we get the following contradiction

$$
0=\left\langle I_{\mu}^{\prime}(u), u\right\rangle=\left(\mu_{1}-\mu\right) \int_{\mathbb{R}^{3}} u^{2} d x-\int_{\mathbb{R}^{3}}|u|^{p+1} d x<0 .
$$

Then we consider the following minimization problem

$$
c_{1}=\inf \left\{I_{\mu}(u): u \in \mathcal{N}_{1}\right\} .
$$

It follows from $\mathcal{N}=\mathcal{N}_{1}$ that $c_{0}=c_{1}$. Next, we want to show $c_{1}>0$. For any fixed $u \in \mathcal{N}_{1}$, let $u=u_{1}+u_{2}, u_{1} \in H_{1}, u_{2} \in H_{2}$, where $H_{2}$ denotes the orthogonal complement of $H_{1}$ in $H$. By the definition of $\mathcal{N}_{1}$, we have $u_{2} \neq 0$. Similar to the proof of Proposition 2.3 in [18], we deduce that for $\lambda=0$,

$$
I_{\mu}(u+w)<I_{\mu}(u), \quad \text { for all } w \in\left\{s u+v: s \geq-1, v \in H_{1}\right\} \backslash\{0\} .
$$

Hence, we get

$$
I_{\mu}(u)=\max \left\{I_{\mu}(v): v \in H_{1} \oplus \mathbb{R}^{+} u_{2}\right\} .
$$

Since $u_{2} \neq 0$, there exists $s>0$ such that $\left\|s u_{2}\right\|=\rho_{1}$, where $\rho_{1}>0$ is given by Lemma 2.4. Then by (1.9) and Lemma 2.4 we get

$$
I_{\mu}(u) \geq I_{\mu}\left(s u_{2}\right) \geq \alpha_{1}>0 .
$$

Thus, we have $c_{1}>0$, and then $c_{0}>0$ by $c_{0}=c_{1}$.

In case $\lambda>0$ in (1.4), at least for $\lambda>0$ small enough, it can be seen as a small perturbation at $\lambda=0$. So, it seems natural to look for a ground state of (1.4) with positive energy, that is, to get $c_{0}>0$. Following the argument of [18], we find that (1.8) does not hold for $\lambda>0$. This motivates us to doubt that the ground state of (1.4) with positive energy does not exist. In other words, $c_{0}>0$ may do not hold any more. Another main result of the present paper is to prove that (1.4) has a nonnegative ground state with negative energy.

Theorem 1.2. Let $p \in(3,5), \lambda>0$ and (V1) hold. Then there is a $\delta_{1}(\lambda)>0$ and $\delta_{1}(\lambda) \leq \delta(\lambda)$ such that for any $\mu \in\left(\mu_{1}, \mu_{1}+\delta_{1}(\lambda)\right)$, problem (1.4) has a nonnegative ground state $u_{0, \mu}$ with $I_{\mu}\left(u_{0, \mu}\right)<0$. Moreover, for any sequence $\mu^{(n)} \downarrow \mu_{1}, u_{0, \mu^{(n)}} \rightarrow 0$ strongly in $H$.

To prove Theorem 1.1 and Theorem 1.2, by delicate analysis of the nonlocal term in (1.5) we first observe that the energy functional $I_{\mu}$ satisfies mountain pass geometry for $\mu>\mu_{1}$ and near $\mu_{1}$. Then using Ekeland's variational principle 
we show that (1.4) has a bound state with negative energy, which also means that $c_{0}<0$. Finally we show that $c_{0}>-\infty$ by the condition (V1).

Next, as a comparison, we recall some known results of (1.4) in the case of $\lambda=0$, i.e.

$$
-\Delta u+V(x) u=\mu u+|u|^{p-1} u, \quad u \in H .
$$

Using the positivity of $e_{1}$, we know that problem (1.10) can not possess any nonnegative bound states for $\mu>\mu_{1}$. Furthermore, by the method of Szulkin and Weth [18] we can prove that problem (1.10) has a ground state with positive energy and the ground state must be sign-changing for $\mu>\mu_{1}$. Theorem 1.1 and Theorem 1.2 show a quite different phenomenon for the problem (1.4) in the case of $\lambda>0$ and $\mu>\mu_{1}$. On the other hand, by using the symmetric property of (1.10) and the condition (V1), problem (1.10) may have infinitely many nontrivial bound states for any $\mu \in \mathbb{R}$ and $1<p<5$. Our next theorem shows that problem (1.4) with $\lambda>0$ also possesses infinitely many nontrivial bound states for any $\mu \in \mathbb{R}$ and $p \in(3,5)$.

Theorem 1.3. Let $p \in(3,5), \lambda>0$ and (V1) hold. Then for any $\mu \in \mathbb{R}$, problem (1.4) has infinitely many nontrivial bound states.

REMARK 1.4. We remark here that the condition (V1) was first introduced by Rabinowitz [15], which guarantees the compact embedding of $H \hookrightarrow L^{q}\left(\mathbb{R}^{3}\right)(2 \leq$ $q<6)$. A weaker version has been given by Bartsch and Wang [8]. We emphasize here that all theorems in the present paper will be still true if the condition (V1) is replaced by the weaker condition given by Bartsch and Wang [8].

This paper is organized as follows. In Section 2, we will prove Theorem 1.1. A key step is to prove that the functional $I_{\mu}$ satisfies mountain pass geometry for $\mu$ in a small right neighborhood of $\mu_{1}$. In Section 3, we will study a suitable minimization problem and then use Ekeland variational principle to prove Theorem 1.2. In Section 4, we will use a generalized version of symmetric mountain pass theorem of Rabinowitz [16] to prove Theorem 1.3.

\section{Nonnegative bound state with positive energy}

This section is devoted to the proof of Theorem 1.1. First, we prove a compactness lemma to the functional $I_{\mu}$ on $H$.

Lemma 2.1. Let $p \in(3,5), \lambda, \mu>0$ and (V1) hold. Assume that a sequence $\left(u_{n}\right) \subset H$ satisfies $\left|I_{\mu}\left(u_{n}\right)\right| \leq M<+\infty$ for all $n \in \mathbb{N}$ and $I_{\mu}^{\prime}\left(u_{n}\right) \stackrel{n}{\longrightarrow} 0$, then $\left(u_{n}\right)$ has a strongly convergent subsequence in $H$.

Remark 2.2. In view of Lemma 2.1, we say that $I_{\mu}$ satisfies Palais-Smale ((PS) in short) condition. 
Proof. By compactness of the embedding $H \hookrightarrow L^{q}\left(\mathbb{R}^{3}\right)(2 \leq q<6)$, it is sufficient to show that $\left(u_{n}\right)$ is bounded in $H$. Choosing $\beta \in(1 /(p+1), 1 / 4)$, then for $n$ large enough we have

$$
\begin{aligned}
& M+\left\|u_{n}\right\| \geq I_{\mu}\left(u_{n}\right)-\beta\left\langle I_{\mu}^{\prime}\left(u_{n}\right), u_{n}\right\rangle \\
& \geq\left(\frac{1}{2}-\beta\right)\left\|u_{n}\right\|^{2}-\left(\frac{1}{2}-\beta\right) \mu \int_{\mathbb{R}^{3}} u_{n}^{2} d x+\left(\beta-\frac{1}{p+1}\right) \int_{\mathbb{R}^{3}}\left|u_{n}\right|^{p+1} d x .
\end{aligned}
$$

For $\mu>0$, by (V1) there exists $R(\mu)>0$ such that $V(x) \geq 2 \mu$ for all $|x| \geq R(\mu)$.

Then

$$
\int_{|x| \geq R(\mu)} u_{n}^{2} d x \leq \int_{|x| \geq R(\mu)} \frac{V(x)}{2 \mu} u_{n}^{2} d x \leq \frac{1}{2 \mu}\left\|u_{n}\right\|^{2} .
$$

On the other hand, by Young's inequality we get for any $\varepsilon>0$,

$$
\begin{aligned}
\int_{|x| \leq R(\mu)} u_{n}^{2} d x & \leq C(\mu)\left(\int_{|x| \leq R(\mu)}\left|u_{n}\right|^{p+1} d x\right)^{2 /(p+1)} \\
& \leq \varepsilon \int_{\mathbb{R}^{3}}\left|u_{n}\right|^{p+1} d x+C(\varepsilon, \mu)
\end{aligned}
$$

Taking $\varepsilon=(\beta-1 /(p+1)) /((1 / 2-\beta) \mu)$ in (2.3) and combining (2.1)-(2.3) we deduce that

$$
M+\left\|u_{n}\right\| \geq \frac{1}{2}\left(\frac{1}{2}-\beta\right)\left\|u_{n}\right\|^{2}-C(\beta, \mu) .
$$

Thus, $\left(u_{n}\right)$ is bounded in $H$.

REMARK 2.3. The proof of (2.4) will play a crucial role to show that $c_{0}>$ $-\infty$, where $c_{0}$ is defined by (1.7), see the proof of Theorem 1.2 , step 2 , in Section 3 .

In order to prove Theorem 1.1, we use the following classical mountain pass Lemma due to Ambrosetti and Rabinowitz [3].

Lemma 2.4. Let $E$ be a real Banach space and the functional $I \in C^{1}(E, \mathbb{R})$. Suppose that $I(0)=0$ and

(a) there are constants $\rho, \alpha>0$ such that $\left.I\right|_{\partial B_{\rho}} \geq \alpha$; and

(b) there is $\bar{u} \in E \backslash \overline{B_{\rho}}$ such that $I(\bar{u})<0$.

Let $c$ be defined by

$$
c=\inf _{g \in \Gamma} \max _{u \in g[0,1]} I(u) \quad \text { with } \Gamma=\{g \in C([0,1], E): g(0)=0, g(1)=\bar{u}\} .
$$

If I satisfies the (PS) condition, then I possesses a critical value $c \geq \alpha$.

In view of Lemma 2.1, a key step of applying Lemma 2.4 is to verify that the functional $I_{\mu}$ satisfy mountain pass geometry, i.e. (a) and (b) in Lemma 2.4. In the case of $0<\mu<\mu_{1}$, it is easy to prove that the functional $I_{\mu}$ defined on $H$ 
satisfies mountain pass geometry. However, for $\mu>\mu_{1}$, it is a difficult issue to prove that the mountain pass geometry holds for $I_{\mu}$. We have to analyze the structure of the functional $I_{\mu}$ delicately and find that the competing of the Poisson term $\int_{\mathbb{R}^{3}} \phi_{u} u^{2} d x$ and the nonlinear term $\int_{\mathbb{R}^{3}}|u|^{p+1} d x$ may produce a new phenomenon to the geometric structure of the functional $I_{\mu}$. And we manage to get the mountain pass geometry of the functional $I_{\mu}$ for $\mu$ in a small right neighbourhood of $\mu_{1}$. The more precise statement is the following lemma.

Lemma 2.5. Let $p \in(3,5), \lambda>0$ and (V1) hold. Then we have the following conclusions:

(a) If $0<\mu<\mu_{1}$, then 0 is a local minimum of $I_{\mu}$.

(b) There are positive constants $\delta(\lambda), \rho(\lambda)$ and $\alpha(\lambda)$ such that, for any $\mu \in$ $\left[\mu_{1}, \mu_{1}+\delta(\lambda)\right),\left.I_{\mu}\right|_{\partial B_{\rho(\lambda)}} \geq \alpha(\lambda)$.

(c) There is $\bar{u} \in H^{1}\left(\mathbb{R}^{3}\right)$ with $\|\bar{u}\|>\rho(\lambda)$ such that $I_{\mu}(\bar{u})<0$ for any $\mu>0$.

Proof. (a) For any $u \in H \backslash\{0\}$, from $p \in(3,5), \lambda>0,0<\mu<\mu_{1}$ and the continuity of the Sobolev embedding of $H$ in $L^{p+1}\left(\mathbb{R}^{3}\right)$, we deduce that

$$
\begin{aligned}
I_{\mu}(u) & =\frac{1}{2}\|u\|^{2}+\frac{\lambda}{4} \int_{\mathbb{R}^{3}} \phi_{u} u^{2} d x-\frac{1}{p+1} \int_{\mathbb{R}^{3}}|u|^{p+1} d x-\frac{\mu}{2} \int_{\mathbb{R}^{3}} u^{2} d x \\
& \geq \frac{1}{2}\|u\|^{2}-C\|u\|^{p+1}-\frac{\mu}{2 \mu_{1}}\|u\|^{2}=\|u\|^{2}\left(\frac{1}{2}-\frac{\mu}{2 \mu_{1}}-C\|u\|^{p-1}\right) .
\end{aligned}
$$

Choosing $\rho_{0}=\|u\|$ small enough such that $C \rho_{0}^{p-1} \leq\left(1-\mu / \mu_{1}\right) / 4$, we obtain that

$$
I_{\mu}(u) \geq \frac{1}{4}\left(1-\frac{\mu}{\mu_{1}}\right) \rho_{0}^{2} .
$$

Therefore the conclusion (a) follows.

(b) Our goal is to prove that for any $\lambda>0$, there exist $\rho(\lambda), \alpha(\lambda), \delta(\lambda)>0$ such that for any $\mu_{1} \leq \mu<\mu_{1}+\delta(\lambda)$,

$$
I_{\mu}(u) \geq \alpha(\lambda), \quad \text { for all } u \in H \text { with }\|u\|=\rho(\lambda) .
$$

We first show that for any $\lambda>0$, there exist constants $\rho(\lambda), \alpha(\lambda)>0$ such that

$$
I_{\mu_{1}}(u) \geq \alpha(\lambda), \quad \text { for all } u \in H \text { with }\|u\|=\rho(\lambda) .
$$

Define

$$
F(u)=\int_{\mathbb{R}^{3}} \phi_{u} u^{2} d x \quad \text { for } u \in H .
$$

Then for any $u \in H$, there exists $t=t(u) \in \mathbb{R}$ and $v \in H_{2}$ such that $u=t e_{1}+v$ and $\|u\|^{2}=t^{2}+\|v\|^{2}$. 
By the mean value theorem we have

$$
\begin{aligned}
\left|F(u)-F\left(t e_{1}\right)\right| & \leq\left|\left\langle F^{\prime}\left(t e_{1}+\theta v\right), v\right\rangle\right|, \quad \text { for } \theta \in[0,1] \\
& =4\left|\int_{\mathbb{R}^{3}} \phi_{t e_{1}+\theta v}\left(t e_{1}+\theta v\right) v d x\right| \\
& \leq 4\left\|\phi_{t e_{1}+\theta v}\right\|_{L^{6}}\left\|t e_{1}+\theta v\right\|_{L^{3}}\|v\|_{L^{2}} \\
& \leq C\left\|t e_{1}+\theta v\right\|^{3}\|v\| \leq C\left(|t|^{3}\|v\|+\|v\|^{4}\right) .
\end{aligned}
$$

Therefore,

$$
\begin{aligned}
I_{\mu_{1}}(u)= & \frac{1}{2}\|u\|^{2}-\frac{\mu_{1}}{2} \int_{\mathbb{R}^{3}} u^{2} d x \\
& +\frac{\lambda}{4}\left(F(u)-F\left(t e_{1}\right)+F\left(t e_{1}\right)\right)-\frac{1}{p+1} \int_{\mathbb{R}^{3}}|u|^{p+1} d x \\
\geq & \frac{1}{2}\left(t^{2}+\|v\|^{2}\right)-\frac{1}{2}\left(t^{2}+\frac{\mu_{1}}{\mu_{2}}\|v\|^{2}\right)-C_{1} \lambda\left(|t|^{3}\|v\|+\|v\|^{4}\right) \\
& +\frac{\lambda}{4} t^{4} \int_{\mathbb{R}^{3}} \phi_{e_{1}} e_{1}^{2} d x-C \int_{\mathbb{R}^{3}}\left(\left|t e_{1}\right|^{p+1}+|v|^{p+1}\right) d x \\
= & \frac{1}{2}\left(1-\frac{\mu_{1}}{\mu_{2}}\right)\|v\|^{2}-C_{1} \lambda\left(|t|^{3}\|v\|+\|v\|^{4}\right) \\
& +C_{2} \lambda t^{4}-C_{3}|t|^{p+1}-C_{4}\|v\|^{p+1} .
\end{aligned}
$$

Setting $\varepsilon_{1}=\left(1-\mu_{1} / \mu_{2}\right) /\left(4 C_{1} \lambda\right)$, then

$$
\begin{aligned}
\frac{1}{2}\left(1-\frac{\mu_{1}}{\mu_{2}}\right) & \|v\|^{2}-C_{1} \lambda|t|^{3}\|v\| \\
& \geq \frac{1}{2}\left(1-\frac{\mu_{1}}{\mu_{2}}\right)\|v\|^{2}-C_{1} \lambda\left(\varepsilon_{1}\|v\|^{2}+\frac{1}{\varepsilon_{1}} t^{6}\right) \\
& =\frac{1}{4}\left(1-\frac{\mu_{1}}{\mu_{2}}\right)\|v\|^{2}-C_{5} \lambda^{2} t^{6}
\end{aligned}
$$

Noting that $3<p<5$, for

$$
|t| \leq \min \left\{1,\left(\frac{C_{2} \lambda}{2\left(C_{3}+C_{5} \lambda^{2}\right)}\right)^{1 /(p-3)}\right\} \triangleq \xi,
$$

and

$$
\|v\| \leq \min \left\{1,\left(\frac{\left(1-\mu_{1} / \mu_{2}\right) / 8}{C_{1} \lambda+C_{4}}\right)^{1 / 2}\right\} \triangleq \eta,
$$

we have

$$
-C_{5} \lambda^{2} t^{6}+C_{2} \lambda t^{4}-C_{3}|t|^{p+1} \geq C_{2} \lambda t^{4}-\left(C_{3}+C_{5} \lambda^{2}\right)|t|^{p+1} \geq \frac{C_{2}}{2} \lambda t^{4}
$$


and

$$
\begin{aligned}
\frac{1}{4}\left(1-\frac{\mu_{1}}{\mu_{2}}\right) & \|v\|^{2}-C_{1} \lambda\|v\|^{4}-C_{4}\|v\|^{p+1} \\
& \geq \frac{1}{4}\left(1-\frac{\mu_{1}}{\mu_{2}}\right)\|v\|^{2}-C_{1} \lambda\|v\|^{4}-C_{4}\|v\|^{4} \\
& \geq \frac{1}{8}\left(1-\frac{\mu_{1}}{\mu_{2}}\right)\|v\|^{2} \geq \frac{1}{8}\left(1-\frac{\mu_{1}}{\mu_{2}}\right)\|v\|^{4}
\end{aligned}
$$

Combining (2.8)-(2.11) we get

$$
I_{\mu_{1}}(u) \geq \frac{C_{2}}{2} \lambda t^{4}+\frac{1}{8}\left(1-\frac{\mu_{1}}{\mu_{2}}\right)\|v\|^{4} \geq C(\lambda)\left(t^{2}+\|v\|^{2}\right)^{2}=C(\lambda)\|u\|^{4} .
$$

Let $\rho(\lambda)=\min \{\xi, \eta\}$. Then by (2.12) we see that (2.6) holds.

For $\mu>\mu_{1}$, we have

$$
I_{\mu}(u)=I_{\mu_{1}}(u)-\frac{1}{2}\left(\mu-\mu_{1}\right) \int_{\mathbb{R}^{3}} u^{2} d x \geq I_{\mu_{1}}(u)-\left(\mu-\mu_{1}\right) C\|u\|^{2} .
$$

This and (2.6) imply that there is $\delta(\lambda)>0$ such that (2.5) holds.

(c) Let $e_{0} \in H \backslash\{0\}$ and $s>0$. Then we have that

$$
\begin{aligned}
I_{\mu}\left(s e_{0}\right) & =\frac{s^{2}}{2}\left(\left\|e_{0}\right\|^{2}-\mu \int_{\mathbb{R}^{3}} e_{0}^{2} d x\right)+\frac{\lambda s^{4}}{4} \int_{\mathbb{R}^{3}} \phi_{e_{0}} e_{0}^{2} d x-\frac{s^{p+1}}{p+1} \int_{\mathbb{R}^{3}}\left|e_{0}\right|^{p+1} d x \\
& \leq \frac{s^{2}}{2}\left\|e_{0}\right\|^{2}+\frac{\lambda s^{4}}{4} \int_{\mathbb{R}^{3}} \phi_{e_{0}} e_{0}^{2} d x-\frac{s^{p+1}}{p+1} \int_{\mathbb{R}^{3}}\left|e_{0}\right|^{p+1} d x .
\end{aligned}
$$

Since $\left\|e_{0}\right\|^{2}, \int_{\mathbb{R}^{3}} \phi_{e_{0}} e_{0}^{2} d x$ and $\int_{\mathbb{R}^{3}}\left|e_{0}\right|^{p+1} d x$ are fixed and positive, the fact of $p \in(3,5)$ implies that there exists $s_{0}>0$ such that

$$
\left\|s_{0} e_{0}\right\|>\rho(\lambda) \quad \text { and } \quad I_{\mu}\left(s_{0} e_{0}\right)<0 .
$$

The conclusion (c) follows from choosing $\bar{u}=s_{0} e_{0}$.

Proof of Theorem 1.1. We denote

$$
c_{1, \mu}=\inf _{\gamma \in \Gamma} \max _{t \in[0,1]} I_{\mu}(\gamma(t)) \quad \text { with } \Gamma=\left\{\gamma \in C([0,1], H): \gamma(0)=0, \gamma(1)=s_{0} e_{0}\right\}
$$

By Lemmas 2.1 and 2.5, the mountain pass Lemma 2.4 implies that $c_{1, \mu}$ is a critical value of $I_{\mu}$ and $c_{1, \mu}>0$. The proof of nonnegativity for at least one of the corresponding critical point is inspired by the idea of [1]. In fact, since $I_{\mu}(u)=I_{\mu}(|u|)$ in $H$, for every $n \in \mathbb{N}$, there exists $\gamma_{n} \in \Gamma$ with $\gamma_{n}(t) \geq 0$ (almost everywhere in $\mathbb{R}^{3}$ ) for all $t \in[0,1]$ such that

$$
c_{1, \mu} \leq \max _{t \in[0,1]} I_{\mu}\left(\gamma_{n}(t)\right)<c_{1, \mu}+\frac{1}{n}
$$


Consequently, by means of Ekeland's principle (see for instance [5]), there exists $\gamma_{n}^{*} \in \Gamma$ with the following properties:

$$
\left\{\begin{array}{l}
c_{1, \mu} \leq \max _{t \in[0,1]} I_{\mu}\left(\gamma_{n}^{*}(t)\right) \leq \max _{t \in[0,1]} I_{\mu}\left(\gamma_{n}(t)\right)<c_{1, \mu}+\frac{1}{n} \\
\left.\left.\max _{t \in[0,1]} \| \gamma_{n}(t)\right)-\gamma_{n}^{*}(t)\right) \|<\frac{1}{\sqrt{n}} \\
\text { there exists } t_{n} \in[0,1] \text { such that } z_{n}=\gamma_{n}^{*}\left(t_{n}\right) \text { satisfies: } \\
I_{\mu}\left(z_{n}\right)=\max _{t \in[0,1]} I_{\mu}\left(\gamma_{n}^{*}(t)\right), \text { and }\left\|I_{\mu}^{\prime}\left(z_{n}\right)\right\| \leq \frac{1}{\sqrt{n}} .
\end{array}\right.
$$

By Lemma 2.1 we get a convergent subsequence (still denoted by $\left(z_{n}\right)_{n \in \mathbb{N}}$ ). We may assume that $z_{n} \rightarrow z$ in $H^{1}\left(\mathbb{R}^{3}\right)$ as $n \rightarrow \infty$. On the other hand, by (2.14), we also arrive at $\gamma_{n}\left(t_{n}\right) \rightarrow z$ in $H^{1}\left(\mathbb{R}^{3}\right)$ as $n \rightarrow \infty$. Since $\gamma_{n}(t) \geq 0$, we conclude that $z \geq 0, z \not \equiv 0$ in $\mathbb{R}^{3}$ with $I_{\mu}(z)>0$ and it is a nonnegative bound state of problem (1.4).

Let $u_{1, \mu}$ be the nonnegative bound state given by the above proof, that is,

$$
I_{\mu}^{\prime}\left(u_{1, \mu}\right)=0 \quad \text { and } \quad I_{\mu}\left(u_{1, \mu}\right)=c_{1, \mu} .
$$

We claim that for any sequence $\mu^{(n)} \downarrow \mu_{1}$, there exists $u_{\mu_{1}} \in H$ with $I_{\mu_{1}}^{\prime}\left(u_{\mu_{1}}\right)=0$ and $I_{\mu_{1}}\left(u_{\mu_{1}}\right)>0$, such that $u_{1, \mu(n)} \rightarrow u_{\mu_{1}}$ strongly in $H$. In fact, by the definition of $c_{1, \mu}$ and the proof of Lemma 2.5(c), we deduce that for $n$ large enough,

$$
\begin{aligned}
0<\alpha(\lambda) & \leq c_{1, \mu^{(n)}} \leq \max _{s \geq 0} I_{\mu^{(n)}}\left(s e_{0}\right) \\
& \leq \max _{s \geq 0}\left\{\frac{s^{2}}{2}\left\|e_{0}\right\|^{2}+\frac{\lambda s^{4}}{4} \int_{\mathbb{R}^{3}} \phi_{e_{0}} e_{0}^{2} d x-\frac{s^{p+1}}{p+1} \int_{\mathbb{R}^{3}}\left|e_{0}\right|^{p+1} d x\right\} .
\end{aligned}
$$

Thus, the critical value sequence $\left\{c_{1, \mu^{(n)}}\right\}$ is bounded from above and below. Then by the proof of Lemma 2.1 we see that the claim holds.

\section{Nonnegative ground state with negative energy}

In this section, we prove another main result of this paper, that is, problem (1.4) has a nonnegative ground state with negative energy.

Proof of Theorem 1.2. We divide the proof into three steps.

Step 1. We claim that there exists $w \in H$ such that

$$
I_{\mu}^{\prime}(w)=0 \quad \text { and } \quad I_{\mu}(w)<0 .
$$

For $\rho(\lambda)>0$ given in the proof of Lemma 2.5, we set

$$
c_{2, \mu}=\inf \left\{I_{\mu}(u):\|u\| \leq \rho(\lambda)\right\} .
$$

It is clear that $c_{2, \mu}>-\infty$. 
We will show that $c_{2, \mu}<0$. In fact, since $\mu>\mu_{1}$ we have for $t>0$ small enough,

$$
\begin{aligned}
I_{\mu}\left(t e_{1}\right)= & \frac{t^{2}}{2}\left(\left\|e_{1}\right\|^{2}-\mu \int_{\mathbb{R}^{3}} e_{1}^{2} d x\right) \\
& +\frac{\lambda}{4} t^{4} \int_{\mathbb{R}^{3}} \phi_{e_{1}} e_{1}^{2} d x-\frac{1}{p+1} t^{p+1} \int_{\mathbb{R}^{3}}\left|e_{1}\right|^{p+1} d x \\
= & \frac{t^{2}}{2}\left(1-\frac{\mu}{\mu_{1}}\right)+C \lambda t^{4}-C t^{p+1}<0 .
\end{aligned}
$$

This implies that $c_{2, \mu}<0$. Note that if $\left(u_{n}\right)$ is a minimizing sequence of $c_{2, \mu}$, then $\left(\left|u_{n}\right|\right)$ is also a minimizing of $c_{2, \mu}$. Therefore by (3.1) and Ekeland's variational principle there exists a sequence $\left(u_{n}\right)$ in $H$ and $u_{n} \geq 0$ such that

$$
I_{\mu}\left(u_{n}\right) \stackrel{n}{\longrightarrow} c_{2, \mu} \quad \text { and } \quad I_{\mu}^{\prime}\left(u_{n}\right) \stackrel{n}{\longrightarrow} 0 \text {. }
$$

It follows from Lemma 2.1 that there exists $w \in H$ and $w \geq 0$ such that $I_{\mu}^{\prime}(w)=0$ and $I_{\mu}(w)<0$.

Step 2. We claim that there exists $u_{0, \mu} \in H$ such that

$$
I_{\mu}^{\prime}\left(u_{0, \mu}\right)=0 \quad \text { and } \quad I_{\mu}\left(u_{0, \mu}\right)=c_{0, \mu}<0
$$

where

$$
c_{0, \mu}=\inf \left\{I_{\mu}(u): u \in \mathcal{N}\right\}, \quad \mathcal{N}=\left\{u \in H \backslash\{0\}: I_{\mu}^{\prime}(u)=0\right\} .
$$

By Step 1 we know that $\mathcal{N} \neq \emptyset$ and $c_{0, \mu}<0$. We will show that $c_{0, \mu}>-\infty$. For any $u \in \mathcal{N}$, similar to the proof of (2.4), we have that

$$
I_{\mu}(u)=I_{\mu}(u)-\beta\left\langle I_{\mu}^{\prime}(u), u\right\rangle \geq \frac{1}{2}\left(\frac{1}{2}-\beta\right)\|u\|^{2}-C(\beta, \mu),
$$

where $\beta \in(1 /(p+1), 1 / 4)$. Thus $c_{0, \mu}>-\infty$. Choosing $u_{n} \in \mathcal{N}$ such that $I_{\mu}\left(u_{n}\right) \stackrel{n}{\longrightarrow} c_{0, \mu}$ and $I_{\mu}^{\prime}\left(u_{n}\right) \stackrel{n}{\longrightarrow} 0$, then by Lemma 2.1 there exists $u_{0, \mu} \in H$ such that $I_{\mu}^{\prime}\left(u_{0, \mu}\right)=0$ and $I_{\mu}\left(u_{0, \mu}\right)=c_{0, \mu}$, which means that problem (1.4) has a ground state $u_{0, \mu}$ with $I_{\mu}\left(u_{0, \mu}\right)<0$.

Step 3. We claim that the solutions given by Steps 1 and 2 coincide. The proof is divided into two steps. In the first place, for any $u \neq 0$ and $u$ is a solution of (1.4) with $\mu=\mu_{1}$, we have that

$$
\|u\|^{2}-\mu_{1} \int_{\mathbb{R}^{3}}|u|^{2} d x+\int_{\mathbb{R}^{3}} \phi_{u} u^{2} d x=\int_{\mathbb{R}^{3}}|u|^{p+1} d x,
$$

and hence

$$
I_{\mu_{1}}(u)=\left(\frac{1}{2}-\frac{1}{p+1}\right)\left(\|u\|^{2}-\mu_{1} \int_{\mathbb{R}^{3}}|u|^{2} d x\right)+\left(\frac{1}{4}-\frac{1}{p+1}\right) \int_{\mathbb{R}^{3}} \phi_{u} u^{2} d x .
$$

Since $\|u\|^{2} \geq \mu_{1} \int_{\mathbb{R}^{3}}|u|^{2} d x$ for any $u \in H$,

$$
I_{\mu_{1}}(u) \geq\left(\frac{1}{4}-\frac{1}{p+1}\right) \int_{\mathbb{R}^{3}} \phi_{u} u^{2} d x>0 .
$$


In the second place, let $u_{0, \mu}$ be the ground state given by the above Step 2. For any sequence $\mu^{(n)} \downarrow \mu_{1}$, by the proof of Lemma 2.1 we deduce that $u_{0, \mu^{(n)}}$ must converge to a critical point of $I_{\mu_{1}}$ with non-positive energy, that is, 0 . Hence there is $\delta_{1}(\lambda)>0$ and $\delta_{1}(\lambda) \leq \delta(\lambda)$ such that for $\mu \in\left(\mu_{1}, \mu_{1}+\delta_{1}(\lambda)\right),\left\|u_{0, \mu}\right\|<\rho(\lambda)$, which implies that $c_{0, \mu}=c_{2, \mu}$. Hence we can conclude that $w=u_{0, \mu}$, which is a nonnegative ground state of (1.4) for $\mu \in\left(\mu_{1}, \mu_{1}+\delta_{1}(\lambda)\right)$. The proof of Theorem 1.2 is complete.

Remark 3.1. (a) Combining Theorems 1.1 and 1.2, we know that (1.4) has at least two nonnegative bound states $u_{0, \mu}$ and $u_{1, \mu}$ for $\mu \in\left(\mu_{1}, \mu_{1}+\delta_{1}(\lambda)\right)$. Theorem 1.2 implies that the ground state $u_{0, \mu}$ bifurcates from zero solution. But Theorem 1.1 implies that the bound state $u_{1, \mu}$ can not bifurcate from zero solution.

(b) In the proof of Step 2 of Theorem 1.2, we do not use the Nehari manifold method, that is, we do not consider the following minimization problem

$$
c_{0}=\inf \left\{I_{\mu}(u): u \in \mathcal{M}\right\}, \quad \mathcal{M}=\left\{u \in H \backslash\{0\}:\left\langle I_{\mu}^{\prime}(u), u\right\rangle=0\right\} .
$$

This is because for $\mu>\mu_{1}$, we cannot deduce that $0 \notin \partial \mathcal{M}$.

\section{Infinitely many nontrivial bound states}

In this section, we will prove Theorem 1.3 by using the condition (V1) and the fact that the problem (1.4) is symmetric with respect to $u \in H$. We start with the following Theorem 4.1 from Rabinowitz [16].

Theorem 4.1. Let $E$ be an infinite dimensional Banach space and let $I \in$ $C^{1}(E, \mathbb{R})$ be even and $I$ satisfies $(\mathrm{PS})$ conditions. Suppose that $I(0)=0$ and $E=Y \oplus X$, where $Y$ is finite dimensional and I satisfies:

(a) there are constants $\rho, \alpha>0$ such that $\left.I\right|_{\partial B_{\rho} \cap X} \geq \alpha$ and

(b) for each finite dimensional subspace $\widetilde{E} \subset E$, there is an $R=R(\widetilde{E})$ such that $I \leq 0$ on $\widetilde{E} \backslash B_{R(\widetilde{E})}$.

Then I possesses an unbounded sequence of critical values.

We are now in a position to use this theorem to prove that the problem (1.4) has infinitely many nontrivial bound states.

Proof of Theorem 1.3. If $\mu<\mu_{1}$, then we may use standard symmetric mountain pass theorem [3], [16] to get the conclusion. In the following, we may assume without loss of generality that $\mu_{k} \leq \mu<\mu_{k+1}$, where $\mu_{k}$ is the $k$-th eigenvalue of $-\Delta+V$ in $H$. From Lemma 2.1, we know that $I_{\mu}$ satisfies (PS) condition. Clearly $I_{\mu}(0)=0$. Choosing $E=H, Y=\operatorname{span}\left\{e_{1}, \ldots, e_{k}\right\}$ and $X=Y^{\perp}$, we are in a position to verify (a) and (b) of Theorem 4.1. 
(a') For any $u \in X$, since $\mu_{k} \leq \mu<\mu_{k+1}$, we have that

$$
\int_{\mathbb{R}^{3}}\left(|\nabla u|^{2}+V(x) u^{2}\right) d x \geq \mu_{k+1} \int_{\mathbb{R}^{3}} u^{2} d x .
$$

Therefore

$$
\begin{aligned}
I_{\mu}(u) & \geq \frac{1}{2}\left(1-\frac{\mu}{\mu_{k+1}}\right)\|u\|^{2}+\frac{\lambda}{4} \int \phi_{u} u^{2} d x-\frac{C_{p+1}}{p+1}\|u\|^{p+1} \\
& \geq \frac{1}{2}\left(1-\frac{\mu}{\mu_{k+1}}\right)\|u\|^{2}\left(1-\frac{2 \mu_{k+1} C_{p+1}}{(p+1)\left(\mu_{k+1}-\mu\right)}\|u\|^{p-1}\right) .
\end{aligned}
$$

Hence there is a $\rho>0$ such that for $\|u\|=\rho$,

$$
I_{\mu}(u) \geq \frac{1}{4}\left(1-\frac{\mu}{\mu_{k+1}}\right) \rho^{2} .
$$

$\left(\mathrm{b}^{\prime}\right)$ For each finite dimensional $\widetilde{E} \subset H$ and for any $v \in \widetilde{E}$,

$$
\begin{aligned}
I_{\mu}(v) & =\frac{1}{2}\|v\|^{2}-\frac{\mu}{2} \int v^{2} d x+\frac{\lambda}{4} \int \phi_{v} v^{2} d x-\frac{1}{p+1} \int|v|^{p+1} d x \\
& \leq \frac{1}{2}\|v\|^{2}-\frac{\mu}{2} \int v^{2} d x+\frac{\lambda C}{4}\|v\|^{4}-\frac{1}{p+1} \int|v|^{p+1} d x .
\end{aligned}
$$

Since $\widetilde{E}$ is finite dimensional and $p \in(3,5)$, we see that there is $R:=R(\widetilde{E})$ such that for all $v \in \widetilde{E} \backslash B_{R(\widetilde{E})}, I_{\mu}(v) \leq 0$.

Now using Theorem 4.1 we know that $I_{\mu}$ possesses an unbounded sequence of critical values and hence the problem (1.4) has infinitely many nontrivial bound states.

Acknowledgements. The authors are grateful to the unknown referee for his/her valuable comments and suggestions, especially on Step 3 of the proof of Theorem 1.2 as well as Remark 3.1(a).

\section{REFERENCES}

[1] S. Alama and G. Tarantello, On semilinear elliptic equations with indefinite nonlinearities, Calc. Var. Partial Differential Equations 1 (1993), 439-475.

[2] A. Ambrosetti, On Schrödinger-Poisson systems, Milan J. Math. 76 (2008), 257-274.

[3] A. Ambrosetti and P.H. Rabinowitz, Dual variational methods in critical point theory and applications, J. Funct. Anal. 14 (1973), 349-381.

[4] A. Ambrosetti and D. Ruiz, Multiple bound states for the Schrödinger-Poisson problem, Commun. Contemp. Math. 10 (2008), 391-404.

[5] T. Aubin And I. Ekeland, Applied Nonlinear Analysis, Wiley, New York, 1984.

[6] A. Azzollini, P. D'Avenia And A. Pomponio, On the Schrödinger-Maxwell equations under the effect of a general nonlinear term, Ann. Inst. H. Poincaré Anal. Non Linéaire 27 (2010), 779-791.

[7] A. Azzollini And A. Pomponio, Ground state solutions for the nonlinear SchrödingerMaxwell equations, J. Math. Anal. Appl. 345 (2008), 90-108.

[8] T. BARTSCH AND Z.-Q. WANG, Existence and multiplicity results for some superlinear elliptic problems on $\mathbb{R}^{N}$, Comm. Partial Differential Equations 20 (1995), 1725-1741. 
[9] V. Benci And D. FortunAto, An eigenvalue problem for the Schrödinger-Maxwell equations, Topol. Methods Nonlinear Anal. 11 (1998), 283-293.

[10] T. D'Aprile AND J. WEI, Standing waves in the Maxwell-Schrödinger equation and an optimal configuration problem, Calc. Var. Partial Differential Equations 25 (2006), 105137.

[11] L. HuAng, E.M. Rocha And J. Chen, Two positive solutions of a class of SchrödingerPoisson system with indefinite nonlinearity, J. Differential Equations 255 (2013), 24632483.

[12] G.B. LI, S.J. PENG AND S.S. YAn, Infinitely many positive solutions for the nonlinear Schrödinger-Poisson system, Commun. Contemp. Math. 12 (2010), 1069-1092.

[13] P.A. Markowich, C. Ringhofer And C. Schmeiser, Semiconductor Equations, Springer-Verlag, New York, 1990.

[14] A. Pankov, Periodic nonlinear Schrödinger equation with application to photonic crystals, Milan J. Math. 73 (2005), 259-287.

[15] P. Rabinowitz, On a class of nonlinear Schrödinger equations, Z. Angew. Math. Phys. 43 (1992), 270-291.

[16] _ Minimax Methods in Critical Point Theory with Applications to Differential Equations, CBMS 65 (American Mathematical Society), Providence, 1986.

[17] D. RuIz, The Schrödinger-Poissom equation under the effect of a nonlinear local term, J. Funct. Anal. 237 (2006), 655-674.

[18] A. Szulkin And T. Weth, Ground state solutions for some indefinite variational problems, J. Funct. Anal. 257 (2009), 3802-3822.

[19] Z.P. WANG AND H.S. Zhou, Positive solution for a nonlinear stationary SchrödingerPoisson system in $\mathbb{R}^{3}$, Discrete Contin. Dyn. Syst. 18 (2007), 809-816.

[20] M. Willem, Minimax Theorems, Birkhäuser, Boston, 1996.

JIANQING CHEN

School of Mathematics and Computer Sciences

Fujian Normal University

Fuzhou, 350108, P.R. CHINA

E-mail address: jqchen@fjnu.edu.cn

Zhengping Wang and Xiaoju Zhang

Wuhan Institute of Physics and Mathematics

Chinese Academy of Sciences

P.O. Box 71010

Wuhan 430071, P.R. CHINA

E-mail address: wangzp@wipm.ac.cn 